\title{
Some problems on idomatic partitions and b-colorings of direct products of complete graphs*
}

\author{
Mario Valencia-Pabon ${ }^{\dagger}$
}

\begin{abstract}
In this note, we deal with the characterization of the idomatic partitions and b-colorings of direct products of complete graphs. We recall some known results on idomatic partitions of direct products of complete graphs and we present new results concerning the b-colorings of the direct product of two complete graphs. Finally, some open problems are given.
\end{abstract}

Keywords: direct product, complete graphs, idomatic partitions, bcolorings.

\section{Introduction and preliminary results}

Let $G=(V, E)$ be an undirected finite simple graph without loops (see reference [3] for classical concepts in graph theory). A set $S \subseteq V$ is called a dominating set if for every vertex $v \in V \backslash S$ there exists a vertex $u \in S$ such that $u$ is adjacent to $v$. A set $S \subseteq V$ is called independent if no two vertices in $S$ are adjacent. A set $S \subseteq V$ is called an independent dominating set of $G$ if it is both independent and dominating. A partition of the vertex set $V$ into independent dominating sets is called an idomatic partition of $G$ $[1,2]$. The maximum size of an idomatic partition of $G$ is called the idomatic number $i d(G)$. An idomatic partition of a graph $G$ into $k$ parts is called an idomatic $k$-partition of $G$. Notice that not every graph has an idomatic $k$-partition, for any $k$. For example, the cycle graph on five vertices $C_{5}$ has no idomatic $k$-partition for any $k$. A proper coloring (coloring for short) of

\footnotetext{
${ }^{*}$ This work is supported by the Math-AmSud Projet 10MATH-04 (France-ArgentineBrazil).

${ }^{\dagger}$ Laboratoire d'Informatique de l'Université Paris-Nord (LIPN), 99 Av. J.-B. Clément, 93430 Villetaneuse, France. E-mail : valencia@lipn.univ-paris13.fr
} 
$G$ is an assignment of colors to the vertices of $G$ such that adjacent vertices are assigned different colors. A $k$-coloring of $G$ is a coloring using exactly $k$ different colors. The smallest number $k$ such that $G$ admits a $k$-coloring is called the chromatic number of $G$ and is denoted by $\chi(G)$. Given a $k$ coloring of $G$, a vertex $v$ is said to be dominant if $v$ is adjacent to at least one vertex receiving each of the $k-1$ colors not assigned to $v$. As remarked by Dunbar et al. in [4], an idomatic partition of a graph $G$ represents a proper coloring of the vertices of $G$ where all vertices are dominant. A $b$-coloring of $G[7]$ is a coloring such that every color class admits at least one dominant vertex. So, b-colorings are relaxed versions of idomatic partitions. Notice that every coloring of $G$ with $\chi(G)$ colors is a b-coloring. The b-chromatic number of $G$, denoted by $\chi_{b}(G)$, is the maximum number $k$ such that $G$ admits a b-coloring with $k$ colors.

The direct product $G \times H$ of two graphs $G$ and $H$ is defined by $V(G \times H)=$ $V(G) \times V(H)$, and where two vertices $\left(u_{1}, u_{2}\right),\left(v_{1}, v_{2}\right)$ are joined by an edge in $E(G \times H)$ if $\left\{u_{1}, v_{1}\right\} \in E(G)$ and $\left\{u_{2}, v_{2}\right\} \in E(H)$. This product is commutative and associative in a natural way (see reference [6] for a detailed description on product graphs).

Let $n$ be a positive integer. We denote by $[n]$ the set $\{1, \ldots, n\}$. The complete graph $K_{n}$ will usually be on the vertex set $[n]$.

Idomatic partitions of graphs were studied in [4] as a special coloring problem on graphs defined as fall colorings. In that work, the authors show the following result.

Theorem 1 ([4]). Let $n_{1}>1$ and $n_{2}>1$ be two integers. The direct product graph $K_{n_{1}} \times K_{n_{2}}$ admits an idomatic $n_{1}$-partition and an idomatic $n_{2}$-partition. Furthermore, if $t>1$ is an integer such that $t \notin\left\{n_{1}, n_{2}\right\}$, then $K_{n_{1}} \times K_{n_{2}}$ has no idomatic t-partition.

Moreover, in [4] it is posed the question of characterizing the idomatic partitions of the direct product of three or more complete graphs. Recently, in [9] it is given a full characterization of the idomatic partitions of the direct product of three complete graphs. By following the same ideas given in [9], in [8] it is given a characterization of the idomatic sets of a direct product of four complete graphs.

The direct product of graphs $G_{1}, G_{2}, \ldots, G_{n}$ will be denoted $\times_{i=1}^{n} G_{i}$. Let $G=\times_{i=1}^{k} K_{n_{i}}$ and let $u=\left(u_{1}, \ldots, u_{k}\right)$ and $v=\left(v_{1}, \ldots, v_{k}\right)$ be vertices of $G$. Then, let

$$
e(u, v)=\left|\left\{i: u_{i}=v_{i}\right\}\right|
$$

be the number of coordinates in which $u$ and $v$ coincide. With this notation we can state that $u$ and $v$ are adjacent in $G=\times_{i=1}^{k} K_{n_{i}}$ if and only if 
$e(u, v)=0$. Therefore, $I \subseteq V(G)$ is independent if and only if $e(u, v)>0$ for any $u, v \in I$. Note also that $e(u, v) \leq k-1$ holds for any $u \neq v$.

Let $X \subset V(G)$, where $G=\times_{i=1}^{k} K_{n_{i}}$, and let

$$
\{e(u, v): u, v \in X \text { and } u \neq v\}=\left\{j_{1}, \ldots, j_{r}\right\} .
$$

Then, we say that $X$ is a $T_{j_{1}, \ldots, j_{r}}$-set.

This note is organized as follows. Let $G=\times_{i=1}^{k} K_{n_{i}}$. In Section 2, we summarize the results concerning independent dominating sets and idomatic partitions of $G$ and we pose two problems. Finally, Section 3 contains some new results concerning b-colorings of the direct product of two complete graphs and an interesting conjecture is posed.

\section{Independent dominating sets and idomatic par- titions in $\times_{i=1}^{k} K_{n_{i}}$}

In this section we give two open problems concerning the independent dominating sets and idomatic partitions in the graph $G=\times_{i=1}^{k} K_{n_{i}}$, with $k \geq 2$ and $n_{i} \geq 2$. We start by rephrasing known results in terms of $T_{j_{1}, \ldots, j_{r}}$-sets.

Notice first that if $G=\times_{i=1}^{2} K_{n_{i}}$, with $n_{i} \geq 2$, and if $I \subseteq V(G)$ is an independent dominating set, then $I$ is a $T_{1}$-set (see [4]). For $k>2$ we have:

Proposition 1 ([9]). Let $G=\times_{i=1}^{3} K_{n_{i}}$, with $n_{i} \geq 2$, and let $I$ be an independent dominating set of $G$. Then, $I$ is either a $T_{1}$-set or a $T_{1,2}$-set.

Moreover, in [9] it is characterized the structure of the independent dominating sets of $\times_{i=1}^{3} K_{n_{i}}$. Such results have been extended to the case $k=4$ as follows :

Proposition 2 ([8]). Let $G=\times_{i=1}^{4} K_{n_{i}}$, with $n_{i} \geq 2$, and let $I$ be an independent dominating set of $G$. Then, $I$ is either a $T_{1}$-set, or a $T_{1,2}$-set or a $T_{1,2,3}$-set

Problem 1. Let $G=\times_{i=1}^{k} K_{n_{i}}$, with $k, n_{i} \geq 2$. Then, for all $i \in[k-1]$, does there exist an independent dominating set of $G$ which is a $T_{1,2, \ldots, i}$-set ?

Notice that problem 1 holds for $k=2,3$ and 4 (see [4, 9, 8]). For $k>4$ this is an open problem.

Let $p r_{i}$ denote the projection homomorphism from $G$ to the $i^{\text {th }}$ factor $K_{n_{i}}$. It is not difficult to deduce that for each $i=1, \ldots, k$, the sets 
$p r_{i}^{-1}(1), \ldots, p r_{i}^{-1}\left(n_{i}\right)$ form an idomatic partition of $G$ into $T_{1,2, \ldots, k-1 \text {-sets }}$ and such partitions are the only idomatic partitions of such type (see [9] for details).

For $k=3$, it has been characterized in [9] the idomatic partitions into idomatic $T_{1}$-sets. Moreover, in [9] it is also proved that there exist idomatic partitions composed of $T_{1}$-sets and $T_{1,2}$-sets, and it is described how to construct such partitions. Therefore, from the total characterization of the idomatic partitions of the graph $G=\times_{i=1}^{3} K_{n_{i}}$, the idomatic number of $G$ can be easily deduced.

For $k=4$, it has been characterized in [8] the idomatic partitions into idomatic $T_{1}$-sets and an example of idomatic partition into idomatic $T_{1,2^{-}}$ sets is given. However, it is not known whether there are idomatic partitions formed by various types of idomatic sets.

Problem 2. For $k \geq 4$, a complete characterization of the idomatic partitions of the graph $\times_{i=1}^{k} K_{n_{i}}$ is still open.

\section{3 b-colorings of $\times_{i=1}^{k} K_{n_{i}}$}

In this section we study the b-colorings of the graph $K_{n} \times K_{m}$, with $2 \leq$ $n \leq m$. The main result of this section is the following theorem.

Theorem 2. Let $G=K_{n} \times K_{m}$, with $2 \leq n \leq m$. Let $\Phi$ be a coloring of $G$. Thus, $\Phi$ is a b-coloring of $G$ if and only if $\Phi$ induces an idomatic partition of $G$.

As a consequence of Theorem 2, we have that $G$ has only two b-colorings corresponding to the only two idomatic partitions of $G$, one with $n$ colors and the other with $m$ colors. Therefore, the b-chromatic number of $G$ is equal to $m$.

It is clear that any idomatic partition of $G$ is in fact a b-coloring of $G$. In order to prove the converse statement, we will prove the following lemmas.

Lemma 1. Let $G=K_{n} \times K_{m}$ with $2 \leq n \leq m$. Assume that $G$ is b-colored and that vertices $(i, j)$ and $(i, t)$ are dominant vertices for different colors a and $b$ respectively, where $i \in[n]$ and $j, t \in[m]$ with $j \neq t$. Then, for any $k \in[n]$, with $k \neq i$, there exists no dominant vertex $(k, s)$, with $s \in\{j, t\}$, for a color $c \notin\{a, b\}$.

Proof. Let $a \neq b$ be the colors of vertices $x=(i, j)$ and $y=(i, t)$ respectively. By hypothesis, $x$ and $y$ are dominant vertices. So, by definition of direct 
product, there exist two vertices $x^{\prime}=\left(i^{\prime}, j\right)$ and $y^{\prime}=\left(i^{\prime \prime}, t\right)$, with $i^{\prime}, i^{\prime \prime} \neq i$, such that vertex $x^{\prime}$ is colored with color $a$ and vertex $y^{\prime}$ is colored with color $b$. Now, assume that there is a dominant vertex $z=(k, j)$, with $k \neq i, i^{\prime}$, colored with a color $c$ different from $a$ and $b$. Clearly, this is impossible because vertex $z$ has no neighbor colored with color $a$. Therefore, $z$ can not be a dominant vertex for the color $c$. In an analogous way we can deduce that no vertex $(k, t)$, with $k \neq i, i^{\prime \prime}$, can be a dominant vertex for a color different from $a$ and $b$.

Lemma 2. Let $G=K_{n} \times K_{m}$ with $2 \leq n \leq m$. Assume that $G$ is b-colored and that vertices $(i, j)$ and $(i, t)$ are dominant vertices for different colors a and $b$ respectively, where $i \in[n]$ and $j, t \in[m]$, with $j \neq t$. For any $k \notin\{j, t\}$ and $i^{\prime}, i^{\prime \prime} \in[n] \backslash\{i\}$, with $i^{\prime} \neq i^{\prime \prime}$, there exist no dominant vertices $\left(i^{\prime}, k\right)$ and $\left(i^{\prime \prime}, k\right)$ for different colors $c$ and $d$ respectively, with $c$ and $d$ different from a and $b$.

Proof. By Lemma 1 , we have that $k \neq j, t$ and $i^{\prime}, i^{\prime \prime} \neq i$. Without loss of generality, assume that $i<i^{\prime}<i^{\prime \prime}$. As in the proof of Lemma 1, we can deduce that there are vertices $\left(s_{1}, j\right),\left(s_{2}, t\right),\left(i^{\prime}, p_{1}\right)$ and $\left(i^{\prime \prime}, p_{2}\right)$ colored with colors $a, b, c$ and $d$ resp. with $s_{1}, s_{2} \neq i$ and $p_{1}, p_{2} \neq k$. Now, consider vertex $(i, k)$. Such a vertex can not be colored with any color in $\{a, b, c, d\}$. Thus, let $e$ be the color of $(i, k)$. Now, as $(i, j)$ is a dominant vertex for color $a$, it must have a neighbor $(u, v)$ colored with color $e$. By construction, $u \neq i$ and $v=k$. Moreover, as $\left(i^{\prime}, k\right)$ is a dominant vertex for color $c$, it must have a neighbor $\left(u^{\prime}, v^{\prime}\right)$ colored with color $e$, with $v^{\prime} \neq k$, which is not possible. An analogous contradiction is obtained for vertices $(i, j),(i, t)$ and $\left(i^{\prime \prime}, t\right)$.

By the commutativity of the direct product, a direct consequence of the previous lemma is the following corollary.

Corollary 1. Let $G=K_{n} \times K_{m}$, with $2 \leq n \leq m$. Then, the b-chromatic number of $G$ is equal to $m$.

Lemma 3. Let $G=K_{n} \times K_{m}$, with $2 \leq n \leq m$. Then, $G$ has only $b$-colorings with $n$ and $m$ colors. Moreover, such b-colorings are always idomatic partitions of $G$.

Proof. Assume that $G$ has a b-coloring with $k$ colors. By Corollary 1, we know that $k \leq m$. Moreover, it is well known that the chromatic number of $G$ is equal to $\min \{n, m\}=n$, and thus $k \geq n$. So, assume first that $n<k<m$. Let $s$ be the minimum number of columns containing at least one dominant vertex for each one of the $k$ color classes. Reordering the 
columns representing $G$, we can assume w.l.o.g. that these are the first $s$ columns. Moreover, by Lemmas 1 and 2, we can reorder the rows in such a way that the first $p$ ones contain at least one dominant vertex for each one of the $k$ colors classes, where $p$ is the smallest positive integer for which this property holds. Moreover, we can assume that the first $t$ rows of these $p$ ones contain at least two dominant vertices of different colors. We consider the following cases :

- Case $t=0$. In this case, by applying Lemma 1 to the columns of $G$, we have that $k$ is at most equal to $n$, which is a contradiction to the definition of $k$.

- Case $t>0$. By Lemmas 1 and 2, we can assume that dominant vertices for different colors in row $i$ occur in consecutive positions, with $i \in$ $\{1, \ldots, t\}$. Suppose that $t=2$ and assume w.l.o.g. that the dominant vertices for different colors in rows 1 and 2 are $(1,1), \ldots,\left(1, u_{0}\right)$ and $\left(2, u_{0}+1\right), \ldots,\left(2, u_{0}+u_{1}\right)$, with $u_{0}, u_{1}>1$ respectively. Now, by using arguments as in the proof of Lemma 1, we know that for each vertex $(1, h)$, with $1 \leq h \leq u_{0}$, there exists at least one vertex $(r, h)$, with $r \neq 1$, having the same color as $(1, h)$. Therefore, the color of vertex $\left(1, u_{0}+w\right)$, with $1 \leq w \leq u_{1}$, must be equal to the color assigned to vertex $\left(2, u_{0}+w\right)$. Otherwise, there is a contradiction to the assumption that dominant vertices in row 1 occur in consecutive positions. Moreover, each vertex $\left(1, u_{0}+w\right)$ is a dominant vertex as $\left(2, u_{0}+w\right)$ which is a contradiction to the minimality of $p$. Therefore, we have $t=1$. By repeating the previous reasoning, we can deduce that $p$ must be equal to 1 . Now, we claim that $s=m$. Suppose that $s<m$ and consider vertex $(1, s+1)$. By construction, such a vertex has at least one neighbor colored with each color in $\{1, \ldots, k\}$, and so, it must be assigned a color not in $\{1, \ldots, k\}$, which is a contradiction to the definition of $k$. So, the only possibility that remains is that $s=m$, and so in all cases there is a contradiction to the definition of $k$. Therefore, $k$ must be equal to $n$ or $m$. Finally, note that each vertex $(1, i)$ is a dominant vertex for the color class $i$, for $i=1, \ldots, m$. So, each column $i$ is colored with color $i$, which is an idomatic partition of $G$ into $m$ idomatic sets.

The same arguments can be used for the rows in order to prove that any b-coloring of $G$ with $n$ colors is an idomatic partition of $G$ into $n$ idomatic sets, where each row $i$ is colored with color $i$.

We present the main open question of this section as a conjecture. 
Conjecture 1. Let $G=\times_{i=1}^{k} K_{n_{i}}$, with $k, n_{i} \geq 2$. Then, any b-coloring of $G$ is an idomatic partititon of $G$.

\section{References}

[1] E. J. Cockayne and S. T. Hedetniemi. Disjoint independent dominating sets in graphs. Discrete Math., 15:213-222, 1976.

[2] E. J. Cockayne and S. T. Hedetniemi. Towards a theory of domination in graphs. Networks, 7:247-261, 1977.

[3] R. Diestel Graph Theory. Graduate text in mathematics: 173, SpringerVerlag, 2010.

[4] J. E. Dunbar, S. M. Hedetniemi, S. T. Hedetniemi, D. P. Jacobs, J. Knisely, R. C. Laskar and D. F. Rall. Fall colorings of graphs. J. of Comb. Math. and Comb. Comput., 33:257-273, 2000.

[5] T. W. Haynes, S. T. Hedetniemi and P. J. Slater. Fundamentals of Domination in Graphs. Marcel Dekker, New York, 1998.

[6] W. Imrich and S. Klavžar. Product Graphs: Structure and Recognition. Wiley-Interscience Series in Discrete Mathematics and Optimization, 2000 .

[7] R. W. Irving and D. F. Manlove. The b-chromatic number of a graph. Discrete Appl. Math., 91:127-141, 1999.

[8] S. Klavžar and G. Mekiš. Independent dominating sets and idomatic partitions in direct products of four complete graphs. Manuscript, 2010.

[9] M. Valencia-Pabon. Idomatic partitions of direct products of complete graphs. Discrete Math., 310(5):1118-1122, 2010. 